\title{
Sampling Plan Based on Operating Characteristic Curve
}

\author{
Masato Kazusaki \\ Quality Assurance Office Health \& Crop Sciences Sector, Sumitomo Chemical Co., Ltd., Osaka, Japan
}

Email address:

kazusakim@sc.sumitomo-chem.co.jp

To cite this article:

Masato Kazusaki. Sampling Plan Based on Operating Characteristic Curve. Science Journal of Analytical Chemistry. Vol. 6, No. 3, 2018, pp. 21-24. doi: 10.11648/j.sjac.20180603.11

Received: July 19, 2018; Accepted: August 2, 2018; Published: August 29, 2018

\begin{abstract}
In the pharmaceutical industry, bulk raw materials are purchased to manufacture the bulk pharmaceutical active ingredients. Some of these bulk raw materials in the packaging is randomly picked up to determine the quality of them. So, the sampling plan is an essential means of testing in quality inspections to make disposition decision. So far, the square root of $\mathrm{N}$ plus one rule has been employed to provide a simple mathematical way to calculate the number of items to be inspected for quality of raw materials. However, this rule is apparently not devised on the statistical consideration. Now, another sampling plan based on the operating characteristic (OC) curve is established. The OC curve is defined with a sample size and the maximum acceptable number of defective items, describing how well sampling plan discriminates between good and bad lots. This sampling plan is associated with risks such as the producer's risk of incorrected rejection by the consumers and the consumer's risk of incorrect acceptance of the lots with unsatisfied quality. The sampling plan based on the OC curve is exploited to validate the reliability on two levels of quality, such as acceptable quality level (AQL) and lot tolerance percent defective (LTPD). This newly established sampling plan is compared with the principle of the square root of $\mathrm{N}$ plus one rule to demonstrate the effectiveness to distinguish the good lots from bad lots for the plants where the individual packaging of raw materials is usually purchased at the level of less than 50. In the case of the number of the individual packaging is less than or equal to16, the capability of the new sampling procedure based on the OC curve for discrimination of the quality of lots inspected is superior or comparable to the principle of square root of $\mathrm{N}$ plus one rule. This paper describes the reliability and efficacy of the single-sampling plan under the principles of the OC curve.
\end{abstract}

Keywords: Sampling Plan, Operating Characteristic Curve, Acceptable Quality Level, Lot Tolerance Percent Defective, In-Coming Material

\section{Introduction}

Incoming materials for pharmaceutical use are frequently stored in the drums or containers. These are routinely inspected to determine whether the quality of materials in the drums or containers meet the specifications for acceptance. A sample of the products is employed to evaluate the quality of the products that are received from outside sources. A sampling plan indicates the number of items from each lot are to be inspected and the criteria for determining the acceptability of the lot. In this case, one must establish how to choose a test sample from the whole packages of the incoming materials.

Pharmaceutical companies employ the square root of $\mathrm{N}$ plus one rule for determination of sample sizes to be inspected, and many employees in the pharmaceutical company are trained to use this rule as a rule of thumb model. Since the 1920s, the square root of $\mathrm{N}$ plus one rule was semi-formalized as the sampling scheme by the Association of Official Agricultural
Chemists. Square root of $\mathrm{N}$ plus one rule is a simple way to decide to decide the sampling size from a population. [1-3] Despite the lack of theoretical support, this rule has been adopted by the agencies. [4, 5] In many laboratories, the square root of the lot size plus one rule has been taken to determine the sample size for the number of individual packages, such as drums, to inspect the quality of the bulk sample in the packages. However, there is some controversy again over whether this sampling procedure is consistent or statistically valid. [6-9] Recently, Form 483 is issued by the Food \& Drug Administration (FDA) based on the report on the adverse findings from their inspections. Sampling plans have been referred to numerous Form 483's as lacking the statistical justification. Acceptable sampling plan is an inspection procedure used to determine whether to accept or reject incoming materials according to the theoretical consideration based on the statistics. 


\section{Method}

Sampling is an inspecting procedure applied in the statistical quality control. The sample is randomly selected and checked for various characteristics. Ideally, a sampling plan should reject all lots with inferior quality, while accepting all lots with satisfactory quality. However, the sampling originally bases on the selected sample in the lots, there might be a chance of making an incorrect decision.

Two levels of quality should be considered in the design of the sampling plan. This first is the acceptable quality level (AQL). That is the quality level desired by the consumer. The second quality level is the lot tolerance proportion defective (LTPD). This is the worst level that the consumer could tolerate.

The single-sampling plan is a decision rule to accept or reject the lots based on the analytical results of the sample selected in the random sampling. This procedure is to take the sample of size, $n$, and examine them individually. If the number of defect does not exceed the predetermined acceptance number, $c$, the in-coming materials are entirely accepted by the consumer.

\subsection{Operating Characteristic Curve}

The distribution of the number of defectives, $d$, in the random sampling sample of $n$ items is binominal with parameters $n$ and $p$, where $p$ is the fraction of defective per lot. The probability of defectiveness is expressed by the following equation,

$$
P(d)=\frac{n !}{d !(n-d) !} p^{d}(1-p)^{n-d}
$$

Ideally, a sampling plan should reject all bad lots, while accepting good lots. In some cases, we could accept the lot with a few defective items. So, the probability of acceptance is the sum of the probabilities of acceptance of the lots with nonconforming items within the acceptable number, $c$. [10] The probability of acceptance is expressed in the following equation,

$$
P(d \leq c)=\sum_{d=0}^{c} \frac{n !}{d !(n-d) !} p^{d}(1-p)^{n-d}
$$

For designing a sampling plan with an operating characteristic (OC) curve, we must designate the two points such as the probability of acceptance of $1-\alpha$ for lots with fraction defective $p_{1}$, and the probability of acceptance of $\beta$ for lots with fraction defective $p_{2}$. These parameters are expressed by the following equations,

$$
\begin{gathered}
1-\alpha=\sum_{d=0}^{c} \frac{n !}{d !(n-d) !} p_{1}^{d}\left(1-p_{1}\right)^{n-d} \\
\beta=\sum_{d=0}^{c} \frac{n !}{d !(n-d) !} p_{2}^{d}\left(1-p_{2}\right)^{n-d}
\end{gathered}
$$

where, $\alpha$ and $\beta$ are the producer's risk and consumer's risk, and $p_{1}$ is acceptable quality level (AQL), and $p_{2}$ lot tolerance percent defective (LTPD), respectively. The producer's risk is the probability of rejecting good lots with fraction defective percent equal to or less than AQL, and the consumer's risk is the probability of accepting bad lots with fraction defective percent more than LTPD.

The OC curve for a sampling number of 50 and the maximum number of nonconforming items of 2 is shown in Figure 1. The curve is $\mathrm{S}$ shape. According to the OC curve depicted in Figure 1, lots with the percent defective equal to or less than $1.7 \%$ are accepted at least $95 \%$. Lots with percent defective equal or more than $10.4 \%$ are rejected at least $90 \%$. Lots between AQL and LTPD are sometimes accepted and sometimes rejected.

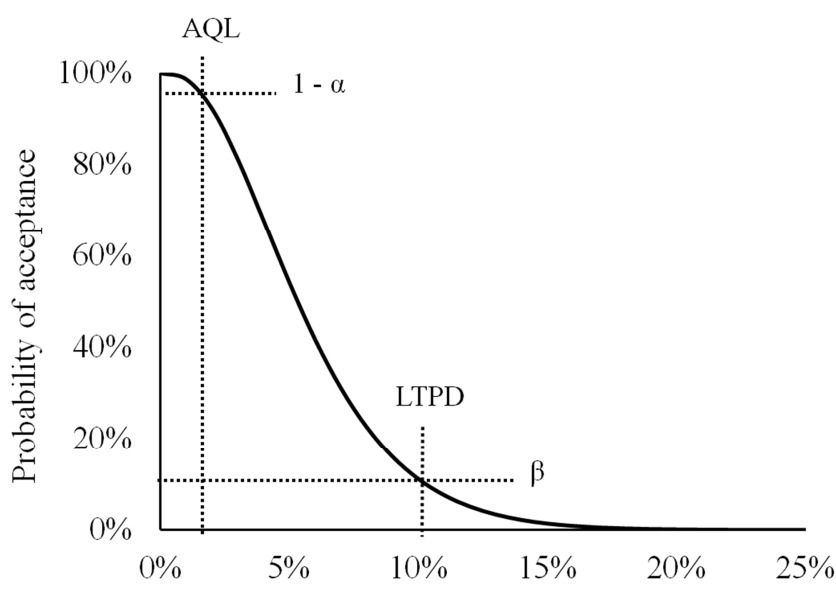

Percent defective

Figure 1. Typical operating characteristic curve for single sampling plan, sampling number is 50 and nonconforming items of 2. Abbreviation; $\alpha$, the producer's risk; $\beta$, the consumer's risk; $A Q L$, acceptable quality level; LTPD, lot tolerance percent defective.

As for raw materials that is employed for the manufacture of the bulk pharmaceutical active ingredients, lots with nonconforming items of zero are accepted traditionally. The OC curve for the sampling number of 5, 10, 20, 50 and the maximum number of nonconforming items of 0 is shown in Figure 2. It is obvious from Figure 2 that the probability of acceptance exponentially decreases with the increase of percent defective. At the same percent defective, the probability of acceptance increases with the decrease in the sampling number.

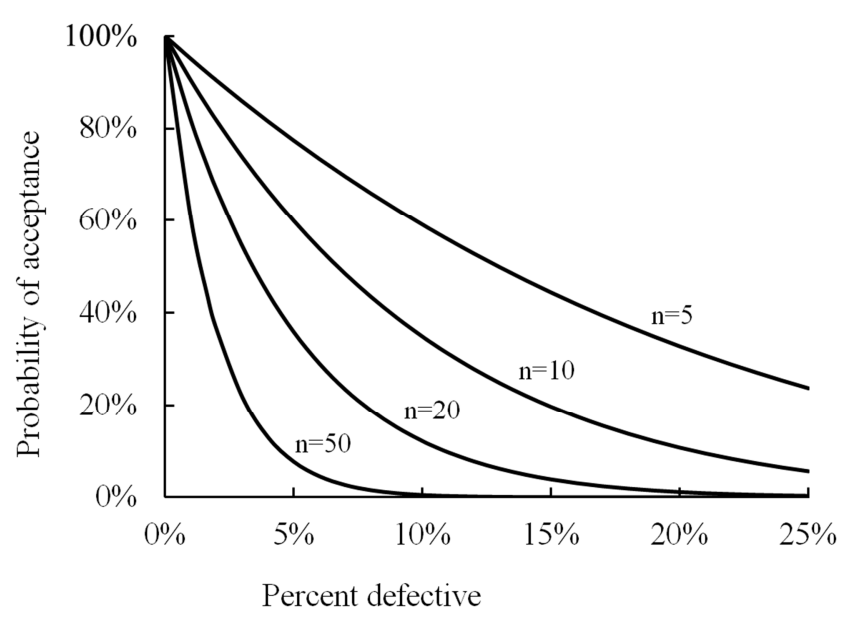

Figure 2. Typical operating characteristic curve for single sampling plan, sampling numbers are 5, 10, 20 and 50, and nonconforming items of 0 . Abbreviation; $\alpha$, the producer's risk; $\beta$, the consumer's risk; $A Q L$, acceptable quality level; LTPD, lot tolerance percent defective. 


\subsection{Discrimination between Good Lots and Bad Lots}

Discrimination is the ability of the sampling plan to distinguish between relatively good levels of quality and relatively bad levels of quality. The high probability of acceptance of $1-\alpha$ is associated with a good level of quality of fraction defective $p_{1}$, and low probability of acceptance of $\beta$ is associated with a bad level of quality of fraction defective $p_{2}$. The discrimination ratio, DR, is defined as

$$
D R=\frac{p_{2}}{p_{1}}
$$

\section{Discussion}

The predominant reason for a sampling is to reduce the fatigue that occurs with $100 \%$ inspection and to effectively minimize the cost of inspections. Many pharmaceutical companies have taken the simple rule, square root of $\mathrm{N}$ plus one, for determining the sample size. However, there is some controversy over whether this sampling plan is statistically valid or not. Now, an OC curve has been used to show how effective the sampling plan is. From the OC curve, we can define the sampling number from the discrimination rate that is driven from AQL and LTPD.

Discrimination ratio below 35 characterizes the sampling plan with a poor capability for the discrimination between good lots and bad lots. [11] Discrimination ratios calculated with the number of nonconforming items of 0 are listed in Table 1, indicating that the discrimination ratio is satisfactory with the sampling number equal or more than 5 . Based on this result, sampling number were established to be 5 with the in-coming raw materials of more than 5 items. With the packaging number of the in-coming raw materials of equal to or less than 5, 100\% inspection should be conducted according to the sampling plan based on the OC curve stated in this paper.

Table 1. Discrimination ratio derived from acceptance quality level and lot tolerance percent defective.

\begin{tabular}{|c|c|c|c|c|c|c|c|}
\hline Sampling number & 4 & 5 & 8 & 10 & 13 & 15 & 20 \\
\hline Discrimination ratio & 34.5 & 36.2 & 39.1 & 40.2 & 41.7 & 41.8 & 42.6 \\
\hline Acceptable quality level (\%) & 1.27 & 1.02 & 0.64 & 0.51 & 0.39 & 0.34 & 0.26 \\
\hline Lot tolerance percent defective $(\%)$ & 43.8 & 36.9 & 25.0 & 20.6 & 16.3 & 14.2 & 10.9 \\
\hline
\end{tabular}

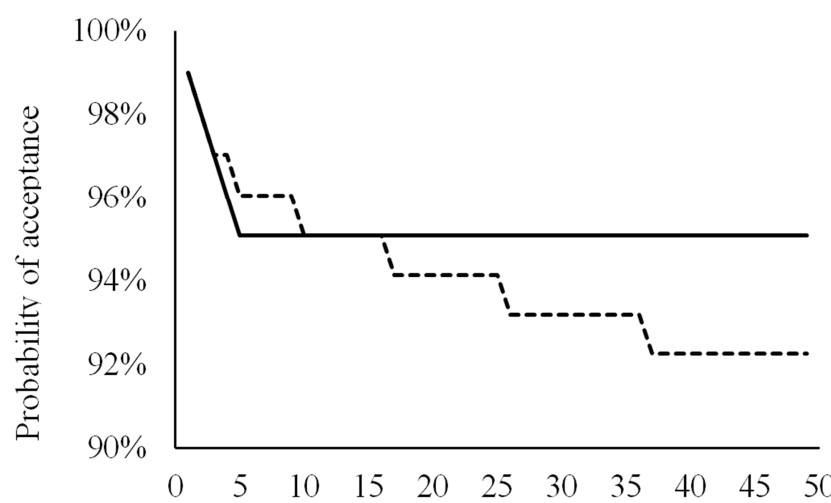

Number of individual packaging of the incoming

Figure 3. Probability of acceptance as a function of the number of individual packaging materials calculated from the sampling plan of square root of $N$ plus one rule and the OC curve. Symbol: solid line, of acceptance derived from the OC curve principle; the dotted line, of acceptance from the square root of $N$ plus one rule.

The probability of acceptance of incoming materials by the OC sampling plan with nonconforming items of 0 is shown for incoming materials with $1.0 \%$ defective in Figure 3 . In the case of the number of the individual packaging is 4 to 9 , the probability of acceptance by the sampling plan from the square root of $\mathrm{N}$ plus one rule is slightly higher than that from the $\mathrm{OC}$ sampling plan, indicating that the sampling plan by a square root of $\mathrm{N}$ plus one rule is less effective to discriminate the bad lots than the $100 \%$ inspection. The number of the individual packaging is 10 to 16 , the probability of acceptance by the OC sampling plan is same to that from the sampling plan of square root of $\mathrm{N}$ plus one rule. In the case that the number of the individual packaging is more than 16 , the probability of acceptance by the OC curve is slightly larger than that by the square root of $\mathrm{N}$ plus one rule. With the increase of the sampling number, the discriminating capability gradually increases for the sampling plan on the square root $\mathrm{N}$ plus one rule. With regards to the incoming materials shipped into our factories, the individual packaging number is always below 10 , indicating the same capability for discrimination between the good lots and the bad lots from the sampling plan on the OC curve and on the principle of square root of $\mathrm{N}$ plus one rule.

\section{Conclusion}

The design of the sampling plan is established from the view point of $\mathrm{OC}$ curve with the consideration of AQL, producer's risk, LTPD and consumer's risk. At the plant for manufacturing the pharmaceutical active ingredients, in-coming ra materials would be rejected if nonconformance was found in the inspection. In this reason, the maximum sampling number is defined to be five from the view point of OC curve.

\section{References}

[1] Morris H. Hansenn, Some History and Reminiscences on Survey Sampling, Statistic Sci, 2, 180 (1987).

[2] D. R. Bellhouse, A Brief History of Survey Sampling Methods, Handbook of Statistics, 6, 1-14 (1998).

[3] Alan Julian Izenman, Statistical and Legal Aspects of the Forensic Study of Illicit Drugs, Statistical Sci, 16, 35 (2001).

[4] FDA, Investigations Operations Manual, Subchapter 4.3: Collection Technique, section 4.3.7.2 Random Sampling.

[5] FDA, CBER. "Draft Points to Consider in the Manufacture of In Vitro Monoclonal Antibodies," March. 1992.

[6] F. W. Quackenbush and R. C. Rund, "The Continuing Problem of Sampling," J. Assoc. Official Analytical Chemists 50, 997 (1967). 
[7] E. M. Foster, "The Control of Salmonellae in Processed Foods: Classification Systems and Sampling Plan,” J. Assoc. Official Analytical Chemists 54, 259 (1971).

[8] Saranadasa, H. 'The Square Root of N Plus One Sampling Rule: How Much Confidence Do We Have?' Pharmaceutical Technology, 27, 50 (2003).

[9] Torbeck L. D. 'Square Root of $(\mathrm{N})+1$ Sampling Plan Is the square root of $(\mathrm{N})+1$ a statistically valid scheme?' Pharmaceutical Technology, 33, 128 (2009).
[10] Arianna Mussidaa, Ursula Gonzales-Barron, Francis Butler, Operating characteristic curves for single, double and multiple fraction defective sampling plans developed for Cronobacter in powder infant formula, Procedia Food Science 1, 979 (2011).

[11] Food and Agriculture Organization of the United Nations, World Health Organization, GENERAL GUIDELINES ON SAMPLING CAC/GL 50-2004. 